\title{
Microwave propagation in air drilling
}

\author{
Meng Xiaofeng ${ }^{1}$, Chen Yijian ${ }^{2}$, Zhou Jing ${ }^{1}$ and Meng Yingfeng ${ }^{2 *}$ \\ ${ }^{1}$ Physics Department, Beijing Normal University, Beijing 100875, China \\ ${ }^{2}$ State Key Laboratory of Oil and Gas Reservoir Geology and Exploitation, Southwest Petroleum University, \\ Chengdu, Sichuan 610500, China
}

(C) China University of Petroleum (Beijing) and Springer-Verlag Berlin Heidelberg 2010

\begin{abstract}
This paper examines the feasibility in air drilling of transmitting down-hole signals by using microwaves. Firstly the basic theory of microwave propagation in the drill-pipe or casing was studied, including power loss, cutoff wavelength, and dust scattering. Theoretical analysis indicates that the microwave propagation distance in a $\Phi 214 \mathrm{~mm}$ casing can easily reach $5,000 \mathrm{~m}$. When the effect of dust particles is taken into account, the propagation distance decreases to $2,000 \mathrm{~m}$. We conducted both laboratory experiments and field tests in casings commonly used in oilfields. The field tests show that the effective propagation distance of microwave in the casing is about 1,300 $\mathrm{m}$. The experimental results do not match well with theoretical prediction, but are acceptable. In future commercial applications, by applying multiple relay amplifiers, the microwave propagation distance could be long enough for most drilling wells.
\end{abstract}

Key words: Microwave, waveguide, drill-pipe, casing, air drilling

\section{Introduction}

In petroleum drilling, signals need to be transmitted from the well-bottom to the surface. These signals include physical characteristics of formation lithology, resident fluids, and reservoirs. Moreover, some geometry properties are included, such as drill bit location, well bore trajectory, etc. Measurement-while-drilling (MWD) is now standard practice in drilling directional wells (Radzinski et al, 2003; Amundsen et al, 2006; Shi, 2006; Seibi et al, 2009). The measurements are made in down-hole and later transmitted to the surface as pressure pulses in the mud system. However, mud-pulse MWD system will be much less efficient if air exists in the mud system and not applicable in a pure air medium. Thus, a new technology called electromagnetic measurements-whiledrilling (EM MWD) has been developed in both USA and Russia (Shale and Moberley, 1992; Soulier and Lemaitre, 1993; Roy and Hay, 1995; Huang, 2003; Li, 2004; Liu et al, 2006). The measured data can be transmitted to the surface antenna receiver through the overlying rock strata using electromagnetic radiation, which is generated by a down-hole transmitter near the drill bit.

Is there any substitute method for EM MWD to transmit signals more efficiently in air drilling? Transmitting microwaves in a waveguide had been widely applied in many fields in our daily life and showed great advantages, such as strong anti-jamming, low energy loss (Miller, 1954; Davidson and Lindsay, 1978). Hence, the State Key Laboratory of Oil

*Corresponding author. email: cwctmyf@vip.sina.com

Received May 6, 2009 and Gas Reservoir Geology and Exploitation in the Southwest Petroleum University propose transmitting measured data in the form of microwaves through the waveguide, which is the drill-pipe or casing in the case of air drilling. In cooperation with the Physics Department in the Beijing Normal University, some theoretical study and laboratory experiments were done. Besides, a model machine was constructed and field tests were conducted.

\section{The microwave power loss in the waveguide}

The key problems in studying microwave propagation characteristics are power loss and noise interference. If the inner surface of the waveguide is smooth enough, noise interference will be eliminated. To simplify the problem, we treated the drill-pipe or casing as an ideal cylinder waveguide, which is fairly smooth, hence this paper emphasizes power loss.

Because the metal drill-pipe or casing is an excellent conductor, the electric field in its side wall is (Guo, 1997):

$$
E(x, t)=E_{0} \exp (-\alpha \cdot x) \exp (i(\beta \cdot x-\omega t))
$$

where $\beta$ is the phase constant, $\mathrm{rad} / \mathrm{m} ; \alpha$ is the attenuation constant, $1 / \mathrm{m} ; \omega$ is the angular frequency, $1 / \mathrm{s} ; t$ is the propagation time, $\mathrm{s} ; x$ is the propagation distance in the radial direction into the side wall, $\mathrm{m}$.

Electromagnetic wave can only propagate into the side wall for a very short distance due to its attenuation (Eq. (1)). Therefore, the conduction current only exists 
in a very thin layer of the inner surface of the waveguide, called surface current. The surface current will dissipate part of electromagnetic energy as Joule heat. Based on the basic theory of EM-wave, the electromagnetic field has an exponential loss in the waveguide along the propagation distance. Supposing that the microwave transmits along the $z$ axis, the electric field can be described as:

$$
E=E_{0}(x, y) \exp (-\alpha z)
$$

where $E_{0}(x, y)$ is the initial electric strength, N/C (Newton/ Coulomb).

Eq. (2) illustrates that the electric field attenuates as $\exp (-\alpha z)$. Similarly, the amplitude of the magnetic field will also attenuate as $\exp (-\alpha z)$. Therefore, the transmission power $P$ should attenuate as $\exp (-2 \alpha z)$. Supposing that the initial power is $P_{0}$, then

$$
P(z)=P_{0} \exp (-2 \alpha z)
$$

In engineering, the attenuation in $\mathrm{dB} / \mathrm{m}, \alpha_{\mathrm{dB}}$ is sometimes used to measure the signal attenuation, instead of the attenuation constant $\alpha$, when signals attenuate rapidly. According to unit conversion relationship, $\alpha_{\mathrm{dB}}$ will be:

$$
\alpha_{\mathrm{dB}}=8.686 \alpha
$$

So, while the signal transmits to distance $z$, the power in decibel is:

$$
P_{\mathrm{dB}}(z)=P_{0, \mathrm{~dB}}-\alpha_{\mathrm{dB}} z
$$

If the initial power of the signal and sensitivity of the detection system are already available, the attenuation constant can be first obtained and then the propagation distance. Or we can solve the maximum attenuation constant according to the desired propagation distance. For example, if the sensitivity of the detection system is $-90 \mathrm{~dB}$, the initial power is $0.24 \mathrm{~W}$, and the expected propagation distance is $5,000 \mathrm{~m}$, the attenuation constant $\alpha_{\mathrm{dB}}$ calculated from Eq. (5) should meet $\alpha_{\mathrm{dB}} \leq 1.676 \times 10^{-2} \mathrm{~dB} / \mathrm{m}$.

\section{Microwave propagation characteristics in the drill-pipe or casing}

Power loss is mainly caused by the surface current and propagation medium in drill-pipe or casing in air drilling.

\subsection{The attenuation constant of different wave modes}

In the previous section, we discussed the relationship between the attenuation constant and power loss (Eq. (5)). However, in some waveguides, multiple wave modes coexist, and their attenuation constants are different from each other. This feature makes power loss in the waveguide more complex. Both transverse electric (TE) and transverse magnetic (TM) wave modes may exist in the cylinder waveguide.

The attenuation constant of $\mathrm{TM}_{n m}$ wave modes is (Ning and Min, 1985):

$$
\alpha_{n m, \mathrm{TM}}=\frac{R_{\mathrm{S}}}{a Z_{0}} \frac{1}{\sqrt{1-\left(\frac{\lambda}{\lambda_{\mathrm{c}, n m}}\right)^{2}}}
$$

$\lambda_{\mathrm{c}, n m}$ is the cutoff wavelength of $\mathrm{TM}_{n m}$, which meets:

$$
\frac{\lambda_{\mathrm{c}, n m}}{a}=\frac{2 \pi}{\rho_{n m}}
$$

where $a$ is the waveguide radius; $Z_{0}=\sqrt{\frac{\mu_{0}}{\varepsilon_{0}}}$ is the wave impedance in free space; $R_{\mathrm{S}}=\sqrt{\frac{\pi f \mu}{\sigma}}$ is the surface resistance of the waveguide; $\mu$ is the waveguide permeability; $\sigma$ is the waveguide conductivity; $f$ is the microwave frequency; $\rho_{n m}$ is the $m$ th root of the Bessel function of $n$th order $J_{n}\left(k_{\mathrm{c}} a\right)=0$, $k_{\mathrm{c}}=2 \pi / \lambda_{\mathrm{c}}$.

The attenuation constant of $\mathrm{TE}_{n m}$ wave modes is:

$$
\alpha_{n m, \mathrm{TE}}=\frac{R_{\mathrm{S}}}{a Z_{0}} \frac{1}{\sqrt{1-\left(\frac{\lambda}{\lambda_{\mathrm{c}, n m}}\right)^{2}}}\left[\left(\frac{\lambda}{\lambda_{\mathrm{c}, n m}}\right)^{2}+\frac{n^{2}}{\left(\rho_{n m}^{\prime}\right)^{2}-n^{2}}\right]
$$

where $\rho_{n m}^{\prime}$ is the $m$ th root of the solution of Bessel function $J_{n}^{\prime}\left(k_{\mathrm{c}} a\right)=0, k_{\mathrm{c}}=2 \pi / \lambda_{\mathrm{c}} ;$ and the cutoff wavelength of $\mathrm{TE}_{n m}$ meets:

$$
\frac{\lambda_{\mathrm{c}, n m}}{a}=\frac{2 \pi}{\rho_{n m}^{\prime}}
$$

The cutoff wavelength is only related to the waveguide radius (Eqs. (7) and (9)). Tables 1 and 2 give the values of $\lambda_{\mathrm{c}, n m} / a$ of several TE and TM wave modes.

Table 1 Values of $\lambda_{\mathrm{c}, n m} / a$ of $\mathrm{TM}_{n m}$ in the cylinder waveguide

\begin{tabular}{ccccc}
\hline & \multicolumn{4}{c}{$\lambda_{\mathrm{c}, n m} / a$} \\
\cline { 2 - 5 } & $n=0$ & $n=1$ & $n=2$ & $n=3$ \\
\hline$m=1$ & $\mathbf{2 . 6 1 2 7}$ & $\mathbf{1 . 6 3 9 8}$ & $\mathbf{1 . 2 2 3 5}$ & 0.9849 \\
$m=2$ & 1.1382 & 0.8956 & 0.7465 & 0.6437 \\
\hline
\end{tabular}

Notes: Data are from Ning and Min, 1985

Table 2 Values of $\lambda_{\mathrm{c}, n m} / a$ of $\mathrm{TE}_{n m}$ in the cylinder waveguide

\begin{tabular}{ccccc}
\hline & \multicolumn{4}{c}{$\lambda_{\mathrm{c}, n m} / a$} \\
\cline { 2 - 5 } & $n=0$ & $n=1$ & $n=2$ & $n=3$ \\
\hline$m=1$ & $\mathbf{1 . 6 3 9 8}$ & $\mathbf{3 . 4 1 2 6}$ & $\mathbf{2 . 0 5 7 2}$ & $\mathbf{1 . 4 9 5 6}$ \\
$m=2$ & 0.8956 & $\mathbf{1 . 1 7 8 5}$ & 0.9369 & 0.7838 \\
$m=3$ & 0.6176 & 0.7361 & 0.6302 & 0.5538 \\
\hline
\end{tabular}

Notes: Data are from Ning and Min, 1985

Taking our field application as example, the microwave frequency and wavelength were $2.4 \mathrm{GHz}$ and $125 \mathrm{~mm}$; and 
the inner radius of the casing was $107 \mathrm{~mm}$ ( $\Phi 214 \mathrm{~mm}$ casing). Thus, $\frac{\lambda}{a} \approx 1.1682$. Since wave modes whose wavelength is shorter than the cutoff wavelength can propagate in the waveguide, only the wave modes which meet $\frac{\lambda_{\mathrm{c}, n m}}{a}>1.1682$ can propagate in drill-pipes and casings (marked in bold italic in Tables 1 and 2).

The conductivity $\sigma$ of steel casings is $5 \times 10^{6} \Omega \cdot \mathrm{m}$, and the magnetic permeability $\mu \approx \mu_{0}$. Substituting them into Eqs. (6) and (8), we can get the attenuation constants of those eight wave modes (Table 3 ).

Table 3 Attenuation constants in $\mathrm{dB} / \mathrm{m}$ of eight wave modes in the $\Phi 214 \mathrm{~mm}$ casing

\begin{tabular}{ccccccccc}
\hline Wave modes & $\mathrm{TM}_{01}$ & $\mathrm{TM}_{11}$ & $\mathrm{TM}_{21}$ & $\mathrm{TE}_{01}$ & $\mathrm{TE}_{11}$ & $\mathrm{TE}_{21}$ & $\mathrm{TE}_{31}$ & $\mathrm{TE}_{12}$ \\
\hline Attenuation constant in $\mathrm{dB} / \mathrm{m}$ & 0.01042 & 0.01303 & 0.03127 & $6.782 \mathrm{E}-3$ & $5.346 \mathrm{E}-3$ & 0.01216 & 0.02519 & 0.07296 \\
\hline
\end{tabular}

Table 3 shows these wave modes attenuate at different rates. Under a specified sensitivity of the detection system, the propagation distance of the mode $\mathrm{TE}_{01}$ may be 10 times that of wave mode $\mathrm{TE}_{12}$ according Eq. (5). If we are able to eliminate the generation of wave modes which have large attenuation constants, the microwave propagation distance will significantly increase.

\subsection{Only one wave mode existing in the drill-pipe or casing}

When there is only one wave mode existing in the pipe, for example $\mathrm{TM}_{01}$, the attenuation constant only relates to the waveguide inner diameter (Eqs. (6) and (7)). Fig. 1 illustrates that the propagation distance increases rapidly with the waveguide inner diameter. Ideally, if the detection sensitivity is $-90 \mathrm{~dB}, \mathrm{TM}_{01}$ wave can easily propagate beyond $5,000 \mathrm{~m}$ in a $\Phi 214 \mathrm{~mm}(r=107 \mathrm{~mm})$ casing (Fig. 1$)$.

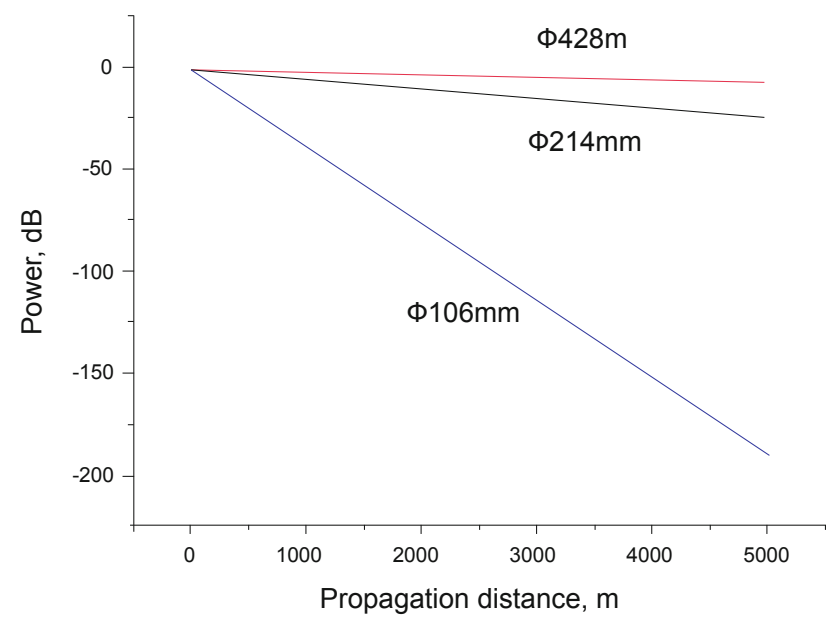

Fig. 1 The relationship between microwave power in decibel and propagation distance for different casings

(Wave mode: $\mathrm{TM}_{01}, f=2.4 \mathrm{GHz} ; P_{0}=600 \mathrm{~mW}$, sensitivity of the detection system: $-90 \mathrm{~dB}$ )

\subsection{Several wave modes coexisting in the drill-pipe or casing}

When several wave modes coexist in the drill-pipe or casing, the propagation distance will increase significantly if the wave modes having larger attenuation constants are restrained (Karbowiak, 1959). In practical cases, the $\mathrm{TE}_{01}$ wave has a relatively small attenuation constant among all wave modes (Albersheim, 1949; Ning and Min, 1985). Besides, $\mathrm{TE}_{01}$ has a distinct surface current distribution, which is a series of circles around the $z$-axis (Fig. 2). In comparison, other wave modes all have surface current along the $z$-axis. As microwave propagation in waveguides be accompanied by a surface current, the wave modes are all restrained except for $\mathrm{TE}_{01}$ if the inner surface of the waveguide is conductive only in the circumferential direction. Fig. 3 is the example of such a waveguide design.

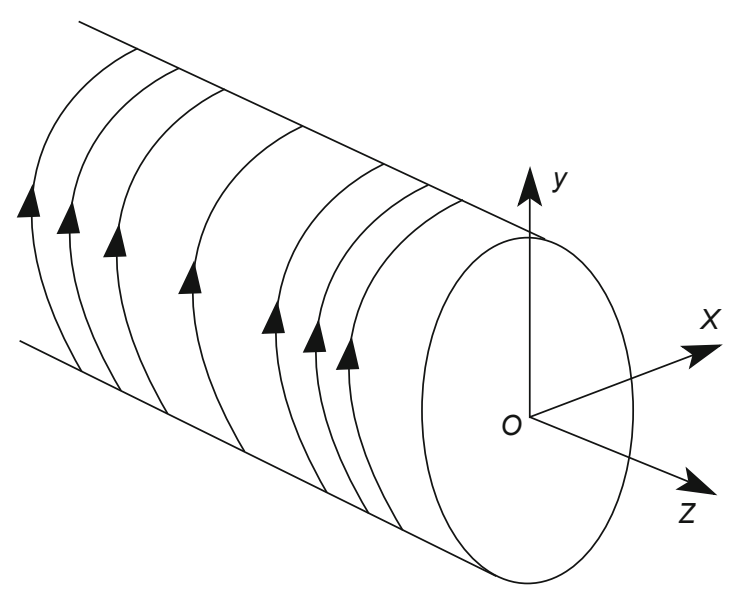

Fig. 2 Surface current distribution of $\mathrm{TE}_{01}$ in the inner surface of the waveguide (after Ning and Min, 1985)

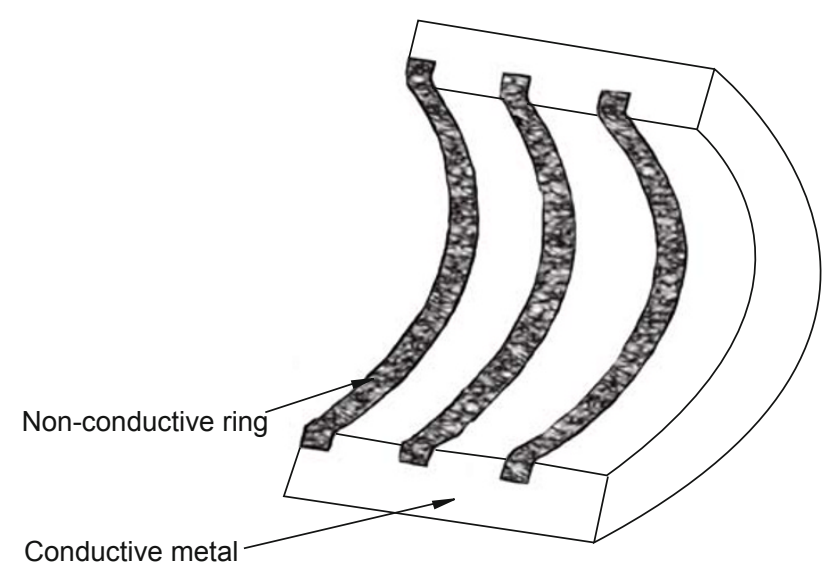

Fig. 3 The specific structure of the inner wall of the waveguide (after Ning and Min, 1985) 


\subsection{Power loss caused by dust in the drill-pipe or casing}

In actual wells, a large amount of dust will exist in the drill-pipe or casing in air drilling. The dust will cause microwave scattering. Therefore, we should take into account of the effect of dust particles when analyzing power loss. Xiong (2000) described in detail the microwave scattering caused by dust particles in air. Here we consider that all incident waves are completely reflected at the surface of the drill-pipe or casing. So, in Section 3.4 we do not take account of the power loss caused by surface current. Our analysis follows the work of Xiong (2000).

The microwave scattering by dust particles is a complex scattering problem for the dust particles of different sizes are randomly oriented. Besides affecting the incident wave, each particle also affects re-scattering or high order scattering wave. In normal air drilling the volume content of dust is very low (less than 1/10000). Most dust particles suspended in the drill-pipe or casing are less than $0.01 \mathrm{~mm}$ in diameter. Only those large dust particles, ranging from $0.01 \mathrm{~mm}$ to 1 $\mathrm{mm}$, are able to scatter incident microwaves and generate rescattering or high order scattering. Since large particles are rare in the drill-pipe or casing, the distance between each other is rather long. Therefore, we can ignore the interaction of the secondary and multiple scattering. Supposing that the single forward scattering is predominant, we simplify dust scattering in the drill-pipe or casing into scattering in a sparse-medium. Therefore, the electric field at the point $(0,0$, $z$ ) can be expressed as follows (Xiong, 2000):

$$
E=E_{0} \exp (-i k z)+E_{0} \frac{S(\theta)}{i k r} \exp (-i k r)
$$

where the first term on the right-hand is the incident field, which is a plane wave propagating along the $z$-axis, with an amplitude of $E_{0}$; the second term is the scattered field; $S(\theta)$ is the scattering amplitude function, related to the particle size and refractive index; $\theta$ is the angle between the incident field (incident direction ) and the scattered field (scattering direction); $r$ is the distance from point $(x, y, 0)$ to point $(0,0, z)$. Only considering the forward scatter, we have

$$
r \cong \frac{x^{2}+y^{2}}{2 z}+z \quad(r>>x, y)
$$

Supposing that 1) The scattered fields generated by the interaction of the incident wave with different dust particles are insignificant; 2) The particles far from the center axis make very little contribution to the scattered field, i.e. $S(\theta) \approx 0$; Eq. (10) can be simplified as follows:

$$
E=E_{\mathrm{i}}\left[1-\frac{2 \pi}{k^{2}} N S(0) L\right]
$$

with

$$
E_{\mathrm{i}}=E_{0} \exp (-i k z)
$$

where $L$ is the microwave propagation distance, $\mathrm{m} ; N$ is the number of randomly distributed particles per unit volume, $1 / \mathrm{m}^{3}$.

The scattered electric field in a uniform medium is:

$$
\begin{aligned}
E & =E_{\mathrm{i}} \exp \left(-i k_{\mathrm{s}} L\right)=E_{0} \exp \left[-i k\left(1+\frac{k_{\mathrm{s}}}{k}\right) L\right] \\
& \cong E_{\mathrm{i}}\left(1-i k_{\mathrm{s}} L\right)
\end{aligned}
$$

where $k_{\mathrm{s}}$ is the propagation constant of scattered wave.

Then, we assume that the dust scattering in the drill-pipe or casing is similar to the scattering in a uniform medium. Now, we can directly solve the attenuation constant (in decibel per meter) by comparing Eqs. (11) and (12):

$$
\alpha_{\mathrm{dB}}=8.686 \frac{2 \pi}{k^{2}} N \operatorname{Re}[S(0)]
$$

When the dust particles have a wide size distribution, the particle-size distribution $N(D)$ should be included into consideration. Eq. (11) can be rewritten as:

$$
E=E_{\mathrm{i}}\left[1-\frac{2 \pi}{k^{2}} L \int_{0}^{D_{\mathrm{m}}} S(0) N(D) \mathrm{d} D\right]
$$

where $N(D)$ is the number of particles in the range of $(D$, $D+\mathrm{d} D$ ), then $N(D) \mathrm{d} D$ is the number of particles of a diameter from $D$ to $D+\mathrm{d} D$ per unit volume; $D_{\mathrm{m}}$ is the maximum diameter.

Thus, the attenuation constant (in $\mathrm{dB} / \mathrm{m}$ ) is:

$$
\alpha_{\mathrm{dB}}=8.686 \frac{2 \pi}{k^{2}} \int_{0}^{D_{m}} \operatorname{Re}[S(0)] N(D) \mathrm{d} D
$$

\subsection{Calculation of the attenuation constant (in $\mathrm{dB} / \mathrm{m}$ )}

We can calculate the attenuation constant (in $\mathrm{dB} / \mathrm{m}$ ) from Eqs. (13) or (15). However, it is very difficult to obtain an accurate particle-size distribution function. The alternative way is assuming that distribution of dust particle size follows approximately log-normal distribution, which occurs widely in nature. Besides, the amplitude of forward scattering field $S(0)$ can be written as (Xiong, 2000):

$$
\begin{aligned}
& S(0)=i x^{3} \frac{\varepsilon-1}{\varepsilon+2} \\
& \operatorname{Re}[S(0)]=-3 x^{3} \frac{\varepsilon^{\prime \prime}}{\left(\varepsilon^{\prime}+2\right)^{2}+\varepsilon^{\prime \prime 2}}
\end{aligned}
$$

where $x=k a ; \varepsilon^{\prime}, \varepsilon^{\prime \prime}$ are the real and imaginary parts of the dielectric constant $\varepsilon$, respectively.

Inserting Eq. (17) into Eq. (15), and letting $D_{\mathrm{m}}=\infty$ gives

$\alpha_{\mathrm{dB}}=0.4288 \times 10^{3} \frac{\varepsilon^{\prime \prime}}{\left(\varepsilon^{\prime}+2\right)^{2}+\varepsilon^{\prime 2}} f N \exp \left(3 m+4.5 \sigma^{2}\right)$

where $\alpha_{\mathrm{dB}}$ is in $\mathrm{dB} / \mathrm{m}, N$ is in $1 / \mathrm{m}^{3}, f$ is in $\mathrm{GHz} ; \varepsilon^{\prime}, \varepsilon^{\prime \prime}, N, m$, and $\sigma$ are related to the number of particles per unit volume, particle size, and water content and their values at drilling sites need to be measured.

For the general situation of air drilling, the value of $\alpha_{\mathrm{dB}}$ is ranging from $10^{-2}$ to $3 \times 10^{-2} \mathrm{~dB} / \mathrm{m}$. When the sensitivity of the detection system is $-90 \mathrm{~dB}$ and the initial power is $0.24 \mathrm{~W}$, the propagation distance decreases from 5,000 to 2,000 m, if considering dust scattering (Eq. 19).

$$
P_{\mathrm{dB}}(z)=P_{0, \mathrm{~dB}}-\left(\alpha_{\text {surface-current, } \mathrm{dB}}+\alpha_{\text {dust, } \mathrm{dB}}\right) z
$$




\section{Laboratory experiments and field application}

\subsection{Laboratory experiments}

In the laboratory, we conducted experiments on four types of casings: three galvanized casings with an inner diameter of 107,82 and $69 \mathrm{~mm}$, respectively, and a rusty casing with an inner diameter of $107 \mathrm{~mm}$. Each casing was $24 \mathrm{~m}$ long. A Rigol DSA1030 spectrum analyzer was used to measure the microwave power from 0.5 to $20.5 \mathrm{~m}$, in a frequency band of 1.4-3.0 GHz. Laboratory apparatus and results are shown in Figs. 4-9. Some wave modes and their cutoff frequencies were marked in Figs. 6 through 9.

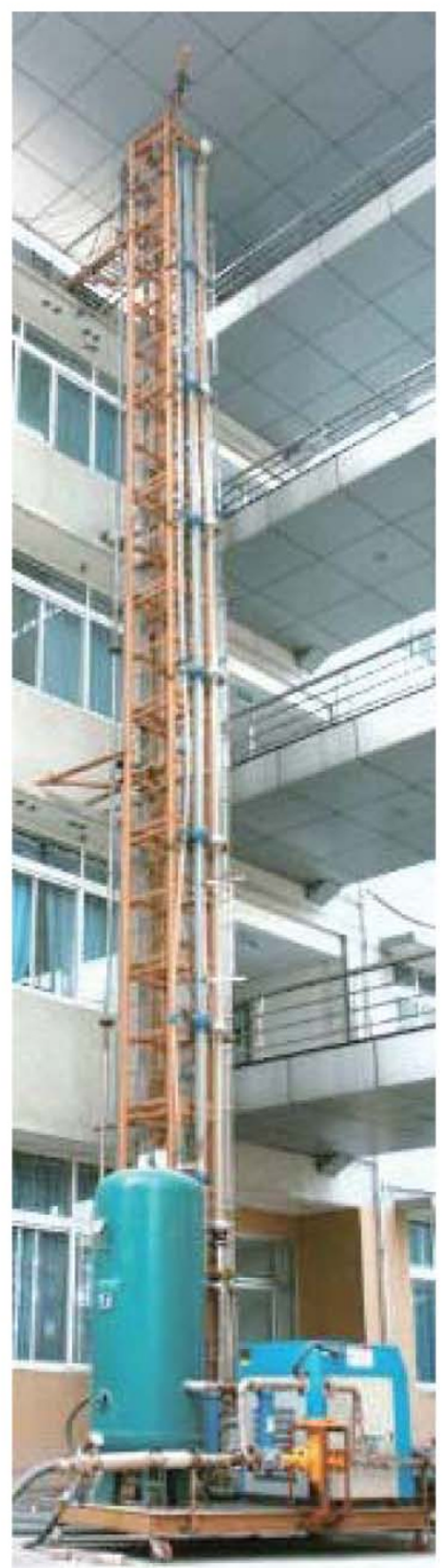

Fig. 4 The derrick model in the laboratory experiments
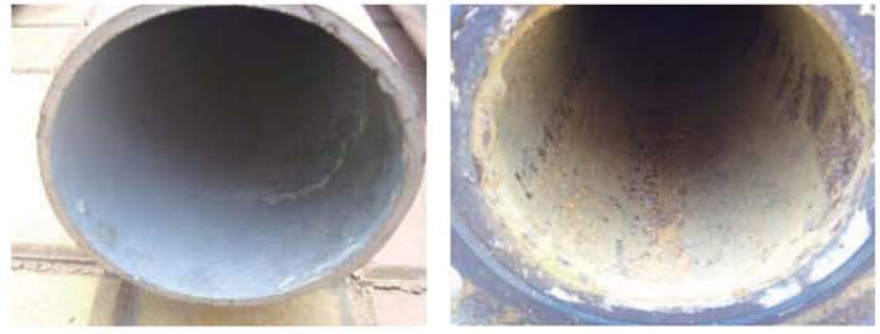

Fig. 5 Inner surface of $\Phi 107 \mathrm{~mm}$ galvanized (left) and rusty (right) casings

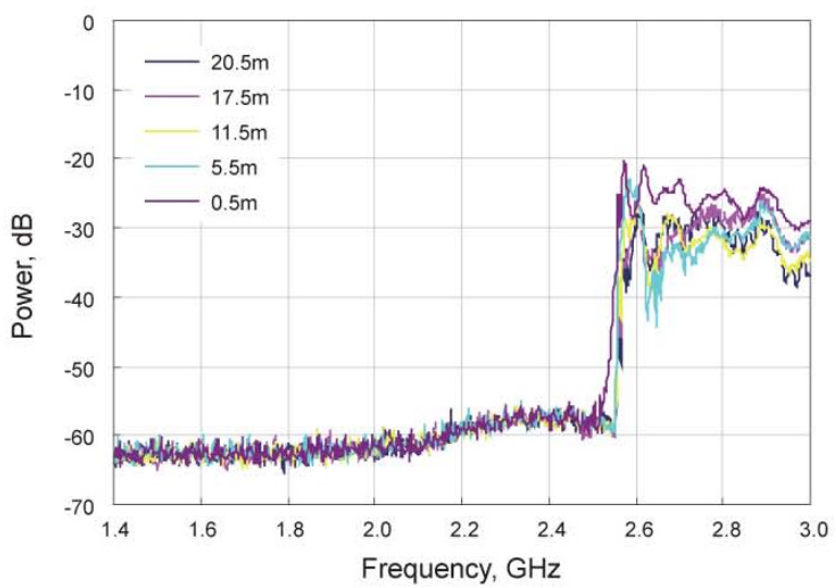

Fig. 6 Laboratory results of $\Phi 69 \mathrm{~mm}$ galvanized casing

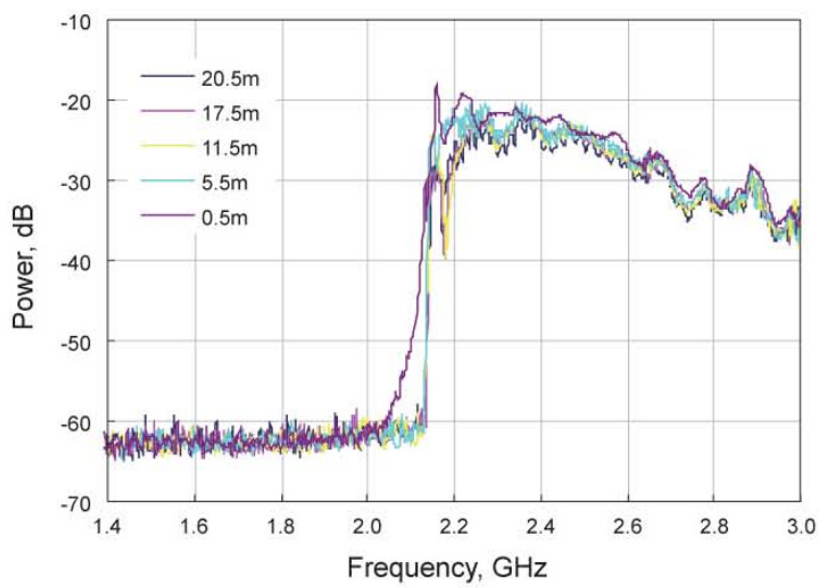

Fig. 7 Laboratory results of $\Phi 82 \mathrm{~mm}$ galvanized casing

In $\Phi 69 \mathrm{~mm}$ galvanized casing, the cutoff frequency for $\mathrm{TE}_{11}$ was $2.55 \mathrm{GHz}$ (Fig. 6). In $\Phi 82 \mathrm{~mm}$ galvanized casing, the cutoff frequency for $\mathrm{TE}_{11}$ and $\mathrm{TM}_{01}$ were 2.15 and 2.8 $\mathrm{GHz}$, respectively (Fig. 7). In $\Phi 107 \mathrm{~mm}$ galvanized casing, the cutoff frequency for $\mathrm{TE}_{11}, \mathrm{TE}_{21}$ and $\mathrm{TM}_{01}$ were 1.64, 2.72, and $2.17 \mathrm{GHz}$, respectively (Fig. 8). In the case of galvanized casings, there existed a distinct cutoff frequency for TE wave modes, but no distinct cutoff frequency for TM wave modes. In the galvanized casings, the power loss in $20 \mathrm{~m}$ was small, no attenuation was observed. However, in the badly rusty casing, the power loss was already very large and the power loss at different distances is significant (Fig. 9). At $20 \mathrm{~m}$, the average power loss was about $30 \mathrm{~dB}$. The reason is that the 
rusty inner surface would cause microwave scattering and dissipate most power into Joule heat. Therefore, keeping the inner surface of drill-pipe or casing from rusting is crucial for microwave propagation.

Moreover, the $\mathrm{TE}_{11}$ wave has a relatively small attenuation

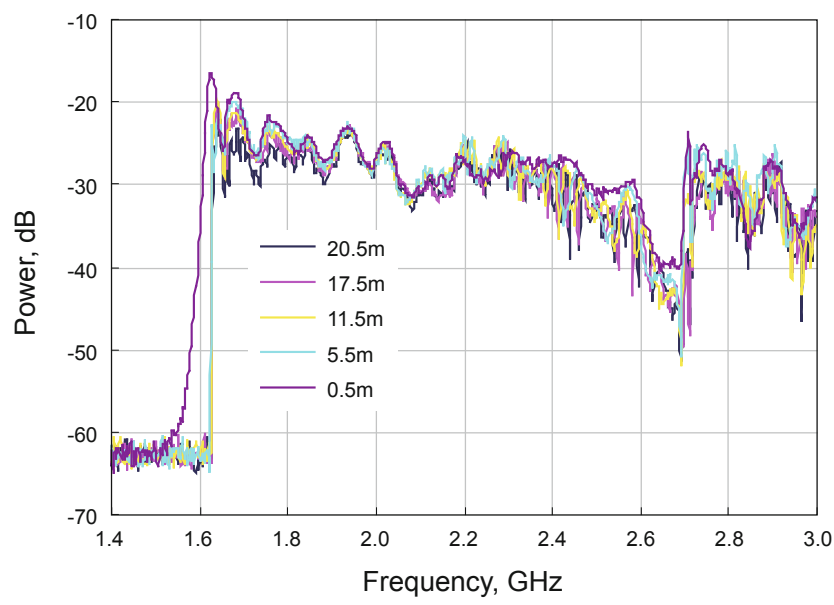

Fig. 8 Laboratory results of $\Phi 107 \mathrm{~mm}$ galvanized casing

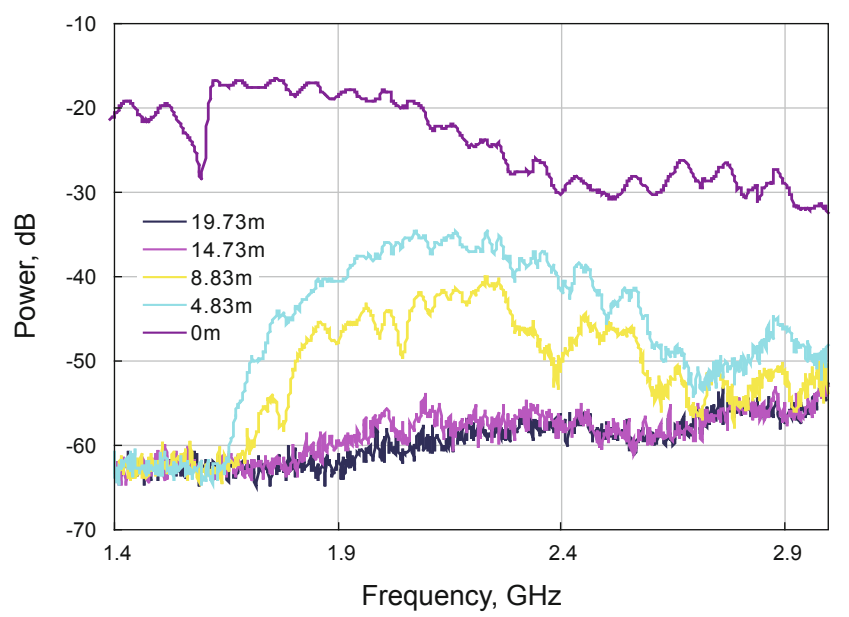

Fig. 9 Laboratory results of $\Phi 107 \mathrm{~mm}$ rusty casing
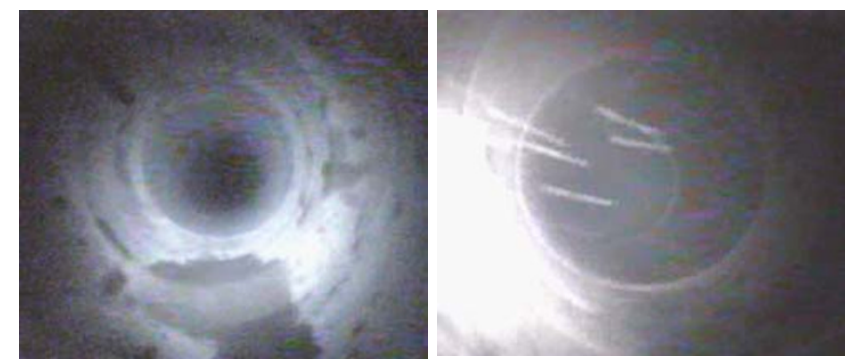

constant among all wave modes. In Fig. 8, when the microwave frequency was smaller than $2.17 \mathrm{GHz}$, only the $\mathrm{TE}_{11}$ wave existed, the microwave power measured at the same distance increased significantly, comparing with in the case of multiple wave modes existing. Experimental results agreed well with the theoretical prediction.

\subsection{Field application}

Two civil frequencies of 2.4 and $5.8 \mathrm{GHz}$ can be used in oil fields. The power generation modules for 5.8 $\mathrm{GHz}$ microwave are too big to install in the drill-pipe or casing; on the other hand, the generation modules for 2.4 $\mathrm{GHz}$ microwave are small. Therefore, we chose $2.4 \mathrm{GHz}$ microwaves to carry out field tests. The model machine was composed of a light source, a camera, and a $2.4 \mathrm{GHz}$ power generation module, which is powered by a battery. We conducted field tests in Xin-3 well, Sichuan Basin. We suspended the model machine down into a $\Phi 214 \mathrm{~mm}$ casing by a steel wire drawer on site and received image signals transmitted by microwave to the surface. The image signals were received fairly clearly till 1,300 m deep, and became blurring at 1,350 $\mathrm{m}$ and absolutely disappeared beyond 1,500 $\mathrm{m}$ (Fig. 10). We observed clearly debris falling into the casing at a depth of $600 \mathrm{~m}$.

The microwave propagation distance measured in field tests is a little shorter than the value calculated from Eq. (19). The possible explanations are as follows: 1) The drill-pipe or casing in Xin-3 well, Sichuan Basin is somewhat rusty, which would cause extra power loss. 2) Some parameters of dust particles used in Eq. (19) are inaccurate for Xin-3 well. 3) The steel wire might disturb the microwave propagation.

Because the microwave propagation distance is limited to around $1,300 \mathrm{~m}$ in field tests, not long enough for most wells, we have to design alternative methods for deep wells: when the microwave propagation distance reaches a limit, a relay amplifier is needed to extend it up to another limit. In this way, the signals can be transmitted to several times longer than $1,300 \mathrm{~m}$. This assumption still needs further investigation.

Fig. 10 Image signals from field tests (From left to right, the well depth is 500, 600, 1000, and 1300 m, respectively)

\section{Conclusions}

Theoretical analysis indicates that it is possible to propagate microwaves in the drill-pipe or casing for a long distance. There are several methods that could extend the propagation distance. 1) If only one wave mode exists in the waveguide, we should extend the inner radius as large as possible. 2) If several wave modes coexist, we can restrain the all wave modes except for the wave mode which has a relatively small attenuation constant by designing specific inner surface structures or choosing a suitable frequency. Moreover, dust in the drill-pipe or casing will surely cause 
power loss. Unfortunately, we can not calculate the accurate value of power loss caused by dust at each well site, lacking measurements of particle-size distribution, water content, and other electric parameters of dust. Instead, we can only conclude that at some well sites, the effective propagation distance will be shortened by more than a half due to dust scattering.

Laboratory results show that a rust-free inner surface is crucial for good power transmission. In field applications we should keep the drill-pipe or casing from rusting. Field tests provide us confidence that transmitting signal by microwave in the drill-pipe or casing is totally applicable, although not as far as the predicted distance. We propose that using relay amplifiers, the propagation distance could be long enough for most wells. The key points of application are choosing appropriate size of drill-pipe or casing, frequency of microwave, and keeping the inner surface rustless.

\section{Acknowledgements}

The authors are grateful for support from the Southwest Engineering Institute of Sinopec and the drilling team of the Xin-3 well.

\section{References}

Albersheim W J. Propagation of $\mathrm{TE}_{01}$ waves in curved wave guides. Bell Syst. Tech. J. 1949. 28: 1-32

Amundsen P, Torkildsen T and Saasen A. Shielding of directional magnetic sensor readings in a measurement while drilling tool for oil well positioning. Journal of Energy Resources Technology. 2006. 128(4): 343-345

Davidson C and Lindsay P. Millimetric waveguide systems: Discussion. Philosophical Transactions of the Royal Society of London. Series A, Mathematical and Physical Sciences. 1978. 289(1356): 123-134

Guo S H. Electrodynamics. Beijing: Higher Education Publishers. 1997 (in Chinese)

Huang M Q. The application of EWR in control of borehole track.
Petroleum Drilling Techniques. 2003. 31(3): 16-18 (in Chinese)

Karbowiak A E. Distortion of information in non-uniform multi-mode waveguides. Macromi Engrs B. 1959. Supplement no.13, 106: 9-16

Li L. The present situation and key technology of EMMWD. China Petroleum Machinery. 2004. 5(32): 53-66 (in Chinese)

Liu X S, Hou X T and Tu Y L. The present and future application of EMMWD. Petroleum Drilling Techniques. 2006. 34(5): 4-9 (in Chinese)

Miller S E. Waveguide as a communication medium. Bell Syst. Tech. J. 1954. 33: 1209-1265

Ning P Z and Min D F. The Technology of Microwave's Transmission. Shanghai: Shanghai Scientific and Technical Publishers. 1985 (in Chinese)

Radzinski P, Mack S, Brady K and Cheatham C. New technology MWD and LWD systems designed for ultra-deepwater drilling. Oil GasEuropean Magazine. 2003. 29(2): 74-80

Roy R and Hay R. Measuring downhole annular pressure while drilling for optimization of underbalanced drilling. Paper presented at the 1995 1st International Underbalanced Drilling Conference, The Hague, The Netherlands, October 2-4

Seibi A, Karrech A, Boukadi F and Pervez T. Wellbore path estimation using measurement while drilling techniques: A comparative study and suggestions for improvements. Energy Sources, Part A: Recovery, Utilization \& Environmental Effects. 2009. 31(14): 12051216

Shale L and Moberley G T. Development of a cartridge data transmission system for use with air drilling motor. Paper SPE/IADC 23907 presented at the 1992 SPE/IADC Drilling Conference, 18-21 February 1992, New Orleans, Louisiana

Shi X F. Methods for orientation for formation layerings and formation boundary prediction with LWD resistivity logs in HZ wells. Well Logging Technology. 2006. 30(2): 119-121 (in Chinese)

Soulier L and Lemaitre M. E.M. MWD data transmission status and perspectives. Paper SPE/IADC 25686 presented at the $1993 \mathrm{SPE} /$ IADC Drilling Conference, 23-25 February 1993, Amsterdam, The Netherlands

Xiong H. Transmission of Radio Wave. Beijing: Publishing House of Electronics Industry. 2000 (in Chinese)

(Edited by Sun Yanhua) 